\title{
Toe brachial index in middle aged patients with diabetes mellitus type 2: Not just a peripheral issue
}

\begin{abstract}
Anna Spångeus, Magnus Wijkman, Torbjörn Lindström, Jan Engvall, Carl Johan Östgren,
\end{abstract} Fredrik H. Nyström and Toste Länne

\section{Linköping University Post Print}

\section{Tweet}

N.B.: When citing this work, cite the original article.

Original Publication:

Anna Spångeus, Magnus Wijkman, Torbjörn Lindström, Jan Engvall, Carl Johan Östgren, Fredrik H. Nyström and Toste Länne, Toe brachial index in middle aged patients with diabetes mellitus type 2: Not just a peripheral issue, 2013, Diabetes Research and Clinical Practice, (100), 2, 195-202.

http://dx.doi.org/10.1016/j.diabres.2013.03.004

Copyright: Elsevier

http://www.elsevier.com/

Postprint available at: Linköping University Electronic Press

http://urn.kb.se/resolve?urn=urn:nbn:se:liu:diva-95976 
Toe Brachial Index in Middle Aged Patients with Diabetes Mellitus Type 2;

-not just a Peripheral Issue

Anna Spångéus ${ }^{1,2}$, Magnus Wijkman², Torbjörn Lindström ${ }^{1,2}$, Jan E Engvall², Carl Johan Östgren $^{2}$, Fredrik H Nystrom ${ }^{1,2}$, Toste Länne ${ }^{2}$

${ }^{1}$ Dept of Endocrinology, County council of Östergötland, Linköping, Sweden, ${ }^{2}$ Dept of Medical and Health Sciences, Linköping University, Sweden

Keywords: diabetes mellitus, toe pressure, toe brachial index, cardiovascular

Competing interests: the authors have no competing interests.

Grants: This study was supported by grants from the Medical Research Council of Southeast Sweden, King Gustav V and Queen Victoria's foundation, the Center for Medical Image Science and Visualization (CMIV), Linköping University, County Council of Östergötland, GE Healthcare, the Swedish Heart-Lung Foundation and the Swedish Research Council Grant 12661 and Diabetes Research Centre.

Word count: 3376

Figures: 3

Tables: 2

Correspondence:

Anna Spångéus MD PhD

Dept of Endocrinology

Linköping University Hospital

58185 Linköping

Sweden

anna.spangeus@liu.se

Phone; +46-010-1030000 


\section{Abstract}

Aim: To explore risk factors for peripheral arterial disease (PAD) as well as the association between toe blood pressure and subclinical and clinical central vascular disease in patients with type 2 diabetes.

Method: Toe Brachial Index (TBI) was cross-sectionally analysed in 742 middle-aged (54-66 years) patients with type 2 diabetes as well as non-diabetic controls and related to other vascular measures (eg carotid intima media thickness (IMT), presence of carotid plaque, central arterial stiffness and left ventricular mass index) and previous cardiovascular events.

Results: A TBI $\leq 0.7$ was seen in $22 \%$ of the patients but only one patient had severe TBI reduction $(\mathrm{TBI} \leq 0.3)$. The corresponding figures in the controls were $13 \%$ and $0 \%$, respectively. Mean TBI was significantly lower in patients with type 2 diabetes than in controls $(0.81 \pm 0.14$ vs. $0.87 \pm 0.15, \mathrm{p}<0.001)$. In patients with diabetes, a lower TBI was associated with increased central arterial stiffness $(p<0.001)$, IMT $(p<0.001)$ and carotid plaque $(\mathrm{p}<0.001)$ as well as with decreasing glomerular filtration rate $(\mathrm{p}<0.001)$. Lower TBI was found in patients with previous macrovascular ischemic events. Furthermore, TBI was negatively correlated with age $(\mathrm{p}<0.001)$, diabetes duration $(\mathrm{p}<0.001)$ and $\mathrm{HbA1c}(\mathrm{p}=0.01)$.

Conclusion: PAD, assessed with TBI, is common in a Swedish middle-aged diabetes type 2 cohort, affecting about one-fifth. As ankle pressure may be confounded by falsely high values in patients with diabetes due to media calcification we conclude that information about TBI may improve the risk evaluation regarding arteriosclerotic disease in both small and large vessels in type 2 diabetes. 


\section{Introduction}

Diabetes mellitus is of increasing concern as the prevalence continues to increase worldwide and is estimated to reach $8 \%$ of the adult population in 2030 [1]. A major health issue is the ensuing increased risk of developing cardiovascular (CV) complications in type 2 diabetes with its consequent risk of increased morbidity and mortality. Ankle brachial index (ABI) has previously been shown to correlate to cardiovascular comorbidity and its' benefits has been highlighted, in addition to the Framingham risk score, in clinical practice [2-4]. Indeed ABI has been pointed out as one of the most feasible screening methods for cardiovascular disease (CVD) since it is easy to perform and also inexpensive [5, 6]. A drawback is the risk of falsely high values due to stiffened, calcified ankle arteries which is mostly found in subjects with diabetes $[3,4,7,8]$. This group, with falsely high $\mathrm{ABI}$ has shown an equal or even higher mortality risk than low ABI why this group is important to detect $[9,10]$. As the arteries of the great toe rarely are involved in the calcification process, assessing toe brachial index (TBI) could provide a more accurate risk evaluation $[3,4,7,8]$.

Peripheral arterial disease (PAD) is common in diabetes mellitus, causing suffering and high costs by ulcerations and chronic pain as well as risk for need of limb amputations.

Furthermore, PAD is associated with increased mortality $[3,11]$. Early interventions are important and can decrease the risk of complications [4]. Taken together, measuring TBI seems important for detecting PAD and might also help assessing general vascular status in subjects with type 2 diabetes.

Health care is mostly publicly financed in Sweden, with a small individual contribution of about 300 USD per year. Routine controls for patients with type 2 diabetes are mainly performed in the primary care. All diabetes care, primary as well as hospital based, adhere to the same national guidelines. It is fundamental to gain more insight on how to best assess the 
risk of different risk factors in subjects with type 2 diabetes since this group of patients have a substantially increased risk for premature cardiovascular morbidity and mortality.

Aortic pulse wave velocity (PWV) measured by tonometry provides a non-invasive estimate of arterial stiffness and is an independent predictive risk factor for all-cause mortality and cardiovascular mortality $[12,13]$. To the best of our knowledge, the association between PWV and TBI in a larger group of patients with type 2 diabetes has previously not been explored. Intima media thickness (IMT) of the carotid arteries measured by B-mode ultrasound is a well known, non-invasive marker of subclinical atherosclerosis and increased risk of CV morbidity $[14,15]$.

Most studies on PAD and CVD risk are based on ABI. The present study focuses on the association between TBI and vascular organ damage and cardiovascular comorbidities reporting data from a large cohort of 742 middle-aged patients with type 2 diabetes compared to data from non-diabetic controls.

\section{Methods}

\section{Settings}

The study was conducted as part of CARDIPP (Cardiovascular Risk factors in Patients with Diabetes - a Prospective study in Primary care) analyzing the baseline data from all patients where toe pressure (TP) measurements were conducted. CARDIPP was launched 2005 to evaluate the prevalence and early risk markers for cardiovascular disease and was designed as an observational prospective study $[16,17]$. The patient enrolment was performed during 2005-2008 and included middle aged (54-66 years) patients with type 2 diabetes, independent of diabetes duration or previous $\mathrm{CV}$ burden, consecutively recruited from 22 different primary health care centres in the counties of Östergötland and Jönköping. Patients with severe medical conditions (e.g. terminal cancer) or severe mental disorder were excluded from 
participation. The catchment area included smaller/middle-sized cities ( $<150.000$ inhabitants) as well as rural areas. As non-diabetic controls, we used age-matched participants from the CAREFUL (Cardiovascular Reference Population) study, i.e. a parallel study to CARDIPP including middle-aged non-diabetic individuals who were subjected to the same study protocol as participants in CARDIPP [18]. The CAREFUL study included randomly selected individuals from a population register aged 50-70 years from the Linköping catchment area. Individuals with known diabetes were excluded as were those with a family history or own diagnosis of aortic aneurysm.

\section{Measures}

All patients were seen on an extended yearly check up at the primary health care centres. In addition, the patients underwent an extensive physiological investigation at the department of Physiology at Linköping University hospital or at Ryhov Hospital (Jönköping), respectively. At the check-up, history of cardiovascular diseases, current medication and life style was recorded.

\section{Laboratory Measures}

Blood samples were obtained in the morning after an overnight fast. The laboratory tests were all analyzed by laboratories at the local primary care centres or hospitals except for apolipoproteins which were analyzed at Linköping University Hospital (Department of Laboratory Medicine). HbA1c was analyzed according to the Swedish Mono-S HPLC standard which is approximately one percent unit lower than the Diabetes Control and Complications Trial (DCCT) standard. Glomerular filtration rate (GFR) was analysed according to Cockcroft-Gault, MDRD as well as cystatinC estimated. In the regression analyses Cockcroft-Gault estimated GFR was used. 


\section{Physiological examination}

Toe pressure (TP) was measured after 10 min rest by strain-gauge technique (Medimatic, Hellerup, Danmark) with the patient in supine position and local temperature at foot level at $30^{\circ} \mathrm{C}$. Measurement was done bilaterally. However, in the present study the lowest recorded TP from each patient was used in the analyses. At the same visit, brachial systolic blood pressure (SBP) was measured bilaterally in supine position by an automated oscillometric device (Dinamap PRO 200 Monitor, Critikon, Tampa, Fl, US) and the arm with the highest pressure was used for further analysis. Simultaneously as TP-measurement a SBP was recorded and used in the subsequent analyses. The toe brachial index (TBI) was calculated as; systolic toe pressure $(\mathrm{mmHg}) /$ systolic brachial pressure $(\mathrm{mmHg})$.

Pulse wave velocity (PWV) in carotid-femoral arteries (PWVcf), i.e. indicating central arterial stiffness, and carotid-radial arteries (PWVcr) was calculated using pulse wave transit time (electrocardiogram-gated recordings of arterial pulse waves in the carotid, radial and femoral artery, respectively) and the distance between the suprasternal notch and the carotid, radial and femoral measurement sites as described in detail previously [16]. A Millar pressure tonometer and the Sphygmo-Cor system (Model MM3, AtCor Medical, Sydney, Australia) were used.

Left ventricular mass (LVM) was measured as described by Devereux [19] with the patient in a left semi-lateral position when performing echocardiography. Basic measurements of the dimensions (left ventricle in diastole and systole, and inter-ventricular septum thickness and posterior wall thickness in diastole) were done in M-mode (The ultrasound scanner was a GE Vivid 7, GE Healthcare, Milwaukee, USA). Left ventricular mass was indexed to body surface area (LVMI) and expressed in $\mathrm{g} / \mathrm{m}^{2}$. 
A B-mode Ultra sound (HDI 5000, ALT,Bothell, Wa, USA) with a broadband linear transducer was used to record intima-media thickness (IMT) of the carotid arteries as described in detail earlier [17]. In brief, three images were captured bilaterally during diastole and transferred to a PC for further analyses. Measurement of IMT was made by manually delimiting the border between lumen/intima as well as media/adventitia in the images. An average of the measurements on the left and the right carotid arteries was used in the further calculations. Following IMT measurement, the presence of carotid plaque was determined. This was done in the carotid bifurcation. A plaque thickness exceeding $1 \mathrm{~mm}$ or $50 \%$ thicker than adjacent segments was considered significant.

\section{Statistics}

IBM SPSS Statistics version 19.0 was used for statistic analysis. Independent t-test was used when comparing means between two groups and ANOVA when comparing means of three groups (Scheffe was used as post hoc test). Pearson correlation was used to test correlation between TBI and vascular, descriptive as well as laborative measures. Logistic and linear regression was applied to further analyse the relation of TBI to aortic PWV, IMT and carotid plaque. P-value $<0.05$ was considered statistically significant.

\section{Ethical}

The study was in line with the principles in the Declaration of Helsinki and was approved by the Regional Ethical Review Board in Linköping. Informed consent was obtained from all participants.

\section{Results}

\section{General}

For patient characteristics see table 1 . In total 742 patients with diabetes mellitus type 2 were included. There were more men $(n=482)$ than women $(n=260)$. Median duration of diabetes 
was 6 years and most of the patients $(77 \%)$ had a duration of less than 10 years. Eighty eight percent of the subjects were overweight or obese as defined by a BMI $>25 \mathrm{~kg} / \mathrm{m}^{2}$, and $56 \%$ had a $\mathrm{BMI} \geq 30 \mathrm{~kg} / \mathrm{m}^{2}$. About one third $(31 \%)$ were prescribed insulin alone or in combination with oral anti-diabetics, $41 \%$ had oral glucose lowering drugs and $28 \%$ were treated with diet only. About two thirds (65\%) were prescribed anti-hypertensive drugs (also including other causes of prescription, i.e. heart failure, micro-albuminuria) while 55\% received lipid lowering medication (statins) and 29\% acetylsalicylic acid (ASA). Eighteen percent of the patients were current smokers, while $49 \%$ were previous smokers and $32 \%$ had never smoked. Swedish moist tobacco was used by $11 \%$ of the patients.

None of the patients had undergone amputation of a lower limb or toe, but two patients had ulcerations in one of the great toes at the physiological examination thus preventing the performance of measurement bilaterally. In these two cases the measurement from the unaffected great toe was used.

A low TBI, i.e. $\leq 0.7$, was seen in $22 \%$ of the patients, but only one $(0.13 \%)$ had a severe reduction $(\mathrm{TBI} \leq 0.3)$. The lowest TBI found was 0.21 and the lowest $\mathrm{TP}$ was $25 \mathrm{mmHg}$. As shown in figure 1, TBI correlated negatively with the duration of diabetes $(r=-0.167$, $\mathrm{p}<0.001)$ and age $(\mathrm{r}=-0.134, \mathrm{p}<0.001)$ and positively with BMI $(\mathrm{r}=0.097, \mathrm{p}=0.009)$. These variables remained independent after correcting for each other. No gender differences were seen (mean TBI for males $0.82 \pm 0.15$ and for females $0.81 \pm 0.13, \mathrm{p}=0.51$ ). Cigarette smoking, but not use of Swedish moist tobacco, was associated with a lower TBI $(p=0.003$ and $p=0.66$, respectively). The TBI was significantly lower in present smokers $(0.78+0.16)$ than in previous smokers $(0.82 \pm 0.13)$ and never-smokers $(0.82 \pm 0.14), p=0.004$ and $p=0.029$, respectively. 


\section{Vascular measurement}

TBI was negatively correlated to IMT $(r=-0.135, p<0.001)$, a higher central arterial pulse wave velocity $(\mathrm{r}=-0.137, \mathrm{p}<0.001)$ and the presence of carotid plaques $(\mathrm{r}=-0.139, \mathrm{p}<0.001)$ (figure 1). Using regression analyses, three different models were composed to establish significant predicting factors:, model 1 included age and diabetes duration, model 2 added previous $\mathrm{CV}$ events (angina, stroke, MI, heart failure and atrial fibrillation) to the factors in model 1 and model 3 added estimated GFR. Independent and significant correlations remained for all three vascular measures i.e. TBI/PWV, TBI/IMT and TBI/carotid plaque (table 2).

No significant correlations were seen between TBI and left ventricular mass index or PWVcr.

\section{Comorbidity and medication}

The prevalence of previous cardiovascular events was relatively low (table 1). Ischemic comorbidity (i.e. stroke, myocardial infarction (MI), previous percutaneous coronary intervention (PCI)/bypass surgeries or angina pectoris as an expression of coronary atherosclerosis) was seen in $15 \%$ of the participants. Figure 2 shows the mean TBI in patients with and without known previous cardiovascular events. Patients who had experienced a previous ischemic event, PCI/bypass-surgery or symptoms of angina pectoris had lower TBI than those without $(p=0.001, p=0.003$ and $p=0.008$, respectively). No significant difference regarding TBI was seen in patients with or without anti-hypertensive drugs in general or when analyzing these separately (i.e. $\beta$-blockers, thiazide diuretics, loop diuretics, ACE-I/ ARB and calcium blockers). Patients treated with insulin alone or combined with oral glucose lowering medication had significantly lower TBI than the patients treated with dietary recommendations only $(0.79 \pm 0.16$ vs. $0.83 \pm 0.14, \mathrm{p}=0.008)$. However, the association disappeared when correcting for disease duration, indicating that duration but not type of treatment was responsible for the significant association. No significant differences were seen 
between patients treated with oral medication $(0.82 \pm 0.13)$ and those on insulin/oral medication or dietary recommendations only $(p=0.095$ and $p=0.49$, respectively). Treatment with acetylsalicylic acid (ASA) and/or statins did not correlate with extent of TBI.

\section{Laboratory data}

Metabolic control, as estimated by a baseline HbA1c, correlated negatively to TBI in patients with diabetes as shown in figure 3 . The association remained statistically significant after adjustment for diabetes duration $(\mathrm{p}=0.032)$. No correlations were found regarding blood lipids measured either as cholesterol/triglycerides or as apolipoproteins. This was also true when adjusting for use of statin (total cholesterol $p=0.20$, triglycerides $p=0.59, \mathrm{HDL}$ cholesterol $p=0.10$, LDL cholesterol $p=0.20$, HDL/LDL $p=0.78$, Apolipoprotein A1 $p=0.72$ and Apolipiprotein B $\mathrm{p}=0.11$ ). GFR was positively correlated with TBI when measured by Cockcroft-Gault GFR $(\mathrm{r}=0.158, \mathrm{p}<0.001)$, cystatinC estimated GFR $(\mathrm{r}=0.118, \mathrm{p}=0.003)$ and only borderline when expressed as MDRD GFR, $(r=0.072, p=0.053)$. Albumin/creatinine ratio did not correlate to TBI $(\mathrm{r}=-0.038, \mathrm{p}=0.312)$. Furthermore plasma potassium correlated negatively with TBI $(r=-0.123, p=0.001)$. The correlation remained after adjusting for GFR as well as for use of anti-hypertensive drugs (drugs analysed separately).

\section{Controls}

As controls, 155 non-diabetic individuals were included. The mean age was as among those with diabetes, i.e. $61 \pm 5$ years $(\mathrm{p}=0.17$, range $52-69$ years). There was a significantly higher proportion of women in the control group, i.e. $52 \%$, compared to $35 \%$ in the diabetes group $(p<0.001)$. Descriptive clinical data in comparison to the patients with type 2 diabetes are summarized in table 1.

Patients with diabetes had significantly lower TBI than controls $(0.81 \pm 0.14$ vs. $0.87 \pm 0.15$, $\mathrm{p}<0.001)$. Thirteen percent of the controls had a TBI $\leq 0.7$, but none had $\mathrm{TBI}<0.3$. In accordance with the observations in the diabetes group, controls showed a negative 
correlation between TBI and age $(\mathrm{r}=-0.28, \mathrm{p}<0.001)$ and IMT $(\mathrm{r}=-0.18, \mathrm{P}=0.03)$ as well as a trend to correlate with central arterial PWV $(\mathrm{r}=-0.147, \mathrm{p}=0.07)$. However, no correlation was found between TBI and BMI or carotid plaque. In contrast to patients with type 2 diabetes there was a gender difference regarding TBI in controls, i.e. TBI was lower in females than in males $(0.85 \pm 0.14$ vs. $0.90 \pm 0.16, p=0.03)$. No significant difference in TBI was seen in controls with or without previous $\mathrm{CV}$ or those with or without antihypertensive drugs, statins or ASA. The levels of blood lipids did not correlate to TBI. Values for GFR were not available for the control group. Linear regression regarding TBI/IMT as well as TBI/central arterial PWV according to model 1 and 2 (as for the patient group) showed decreasing significance (IMT model1 $\mathrm{p}=0.06$ and IMT model $2 \mathrm{p}=0.09$ as well as PVW model1 $\mathrm{p}=0.37$ and PVW model2 $\mathrm{p}=0.10)$.

\section{Discussion}

The present study, focusing on TBI, included a large number of middle-aged patients with type 2 diabetes. Though none of the patients had undergone amputation, $22 \%$ were found to have a TBI below 0.7 in one of the toes, thus indicating PAD $[7,8,20]$ and an increased risk of future need of vascular interventions. TBI was significantly lower in the patients with type 2 diabetes than in the controls. In the control group $13 \%$ had a TBI $<0.7$ which is similar or slightly higher than earlier reports on PAD frequency as measured by ABI [3, 4, 21, 22]. Taken together the present study suggests a higher prevalence of PAD in patients with diabetes than in the general population indicating that about one fifth of middle aged patients with type 2 diabetes have subclinical signs of PAD.

Similar to earlier reports on PAD (mainly evaluated by ABI) $[2,3,5,7,23]$, patients with diabetes with a low TBI were found to have a higher prevalence of ischemic events. In support of these findings, the present study showed an association between peripheral vascular disease, as established by low TBI, and central vascular disease, defined as increased 
central arterial stiffness, elevated IMT and presence of carotid plaques. Central arterial stiffness has previously been associated to PAD assessed by $\mathrm{ABI}$ in the general population [23-25], but has, to the best of our knowledge, not been studied regarding TBI in a larger group of patients with type 2 diabetes. Both central arterial stiffness and IMT was independently negatively connected to TBI. The associations seemed stronger in diabetes than in controls and was independent of diabetes duration, age, previous ischemic events and kidney function, all factors known to influence vascular disease.

PAD, as defined by TBI $<0.64$ (thus slightly lower than in our study), was present in $15 \%$ of the patients in a study by Poulsen et al analysing 305 patients with type 2 diabetes, $>20$ years old, without a history of CVD [26]. Seventy eight percent had, in addition to PAD, objective signs of ischemic heart disease and/or carotid arterial disease (assessed by myocardial perfusion scintigraphy and IMT, respectively). As subjects with a history of previous ischemic events were excluded from the study, this strengthens the observation of an often asymptomatic and generalized disease in patients with type 2 diabetes.

In general, hyperlipidemia is considered to increase the risk of vascular events, including PAD [3, 20]. However, the present study could not find a correlation to TBI even when correcting for lipid lowering drugs, heart failure, diabetes duration as well as for age (data not shown). Similar observations have previously been made in diabetes where PAD was diagnosed by ABI [23].

Smokers had a lower TBI, which is in accordance with earlier findings where PAD was found to be more common in smokers $[3,4,20]$. However, previous smokers did not show a lower TBI nor did the patients using Swedish moist tobacco (non-smokable oral form of tobacco). Patients with diabetes and/or renal insufficiency have a higher risk of PAD and other CV diseases $[3,4,20,23,27,28]$. Accordingly, we found a correlation between low GFR and a low TBI. The association between PAD and albuminuria has previously shown conflicting 
results $[23,29-31]$. Our study could not find any correlation between TBI and the albumin/creatinine ratio.

In conclusion, PAD was present in about one fifth of the patients in an unselected middleaged cohort of patients with type 2 diabetes. This implies a high risk for future need of surgical treatments or the development of foot ulcers. A lower TBI in patients with type 2 diabetes was furthermore associated with subclinical and clinical central arterial disease. The clinical implication from this study is that information on arterial stiffness and subclinical atherosclerosis that goes beyond clinical PAD can be obtained from measuring TBI which is a low-cost, easily available noninvasive method. This may call for a more frequent use of TBI measurement in diabetes care than previous recognised.

\section{Acknowledgement}

This study was supported by grants from the Medical Research Council of Southeast Sweden, King Gustav V and Queen Victoria's foundation, the Center for Medical Image Science and Visualization (CMIV), Linköping University, County Council of Östergötland, GE Healthcare, the Swedish Heart-Lung Foundation and the Swedish Research Council Grant 12661 and Diabetes Research Centre.

\section{References}

1. Shaw, JE, Sicree, RA, Zimmet, PZ. Global estimates of the prevalence of diabetes for 2010 and 2030. Diabetes Res. Clin. Pract., 2010;1:4-14.

2. Ankle Brachial Index Collaboration, Fowkes, FG, Murray, GD, Butcher, I, Heald, CL, Lee, RJ et al. Ankle brachial index combined with Framingham Risk Score to predict cardiovascular events and mortality: a meta-analysis. JAMA, 2008;2:197-208. 
3. Norgren, L, Hiatt, WR, Dormandy, JA, Nehler, MR, Harris, KA, Fowkes, FG et al. InterSociety Consensus for the Management of Peripheral Arterial Disease (TASC II). Eur. J. Vasc. Endovasc. Surg., 2007:S1-75.

4. Stehouwer, CD, Clement, D, Davidson, C, Diehm, C, Elte, JW, Lambert, M et al. Peripheral arterial disease: a growing problem for the internist. Eur. J. Intern. Med., 2009;2:132-138.

5. Criqui, MH, Alberts, MJ, Fowkes, FG, Hirsch, AT, O'Gara, PT, Olin, JW et al. Atherosclerotic Peripheral Vascular Disease Symposium II: screening for atherosclerotic vascular diseases: should nationwide programs be instituted?. Circulation, 2008;25:28302836.

6. Creager, MA, White, CJ, Hiatt, WR, Criqui, MH, Josephs, SC, Alberts, MJ et al. Atherosclerotic Peripheral Vascular Disease Symposium II: executive summary. Circulation, 2008;25:2811-2825.

7. Cao, P, Eckstein, HH, De Rango, P, Setacci, C, Ricco, JB, de Donato, G et al. Chapter II: Diagnostic methods. Eur. J. Vasc. Endovasc. Surg., 2011:S13-32.

8. Aboyans, V, Ho, E, Denenberg, JO, Ho, LA, Natarajan, L, Criqui, MH. The association between elevated ankle systolic pressures and peripheral occlusive arterial disease in diabetic and nondiabetic subjects. J. Vasc. Surg., 2008;5:1197-1203.

9. Suominen, V, Uurto, I, Saarinen, J, Venermo, M, Salenius, J. PAD as a risk factor for mortality among patients with elevated ABI--a clinical study. Eur. J. Vasc. Endovasc. Surg., 2010;3:316-322.

10. Arain, FA, Ye, Z, Bailey, KR, Chen, Q, Liu, G, Leibson, CL et al. Survival in patients with poorly compressible leg arteries. J. Am. Coll. Cardiol., 2012;4:400-407.

11. Morillas, P, Quiles, J, Cordero, A, Guindo, J, Soria, F, Mazon, P et al. Impact of clinical and subclinical peripheral arterial disease in mid-term prognosis of patients with acute coronary syndrome. Am. J. Cardiol., 2009;11:1494-1498.

12. Sutton-Tyrrell, K, Najjar, SS, Boudreau, RM, Venkitachalam, L, Kupelian, V, Simonsick, EM et al. Elevated aortic pulse wave velocity, a marker of arterial stiffness, predicts cardiovascular events in well-functioning older adults. Circulation, 2005;25:3384-3390.

13. Wykretowicz, A, Gerstenberger, P, Guzik, P, Milewska, A, Krauze, T, Adamska, K et al. Arterial stiffness in relation to subclinical atherosclerosis. Eur. J. Clin. Invest., 2009;1:11-16.

14. Brohall, G, Oden, A, Fagerberg, B. Carotid artery intima-media thickness in patients with Type 2 diabetes mellitus and impaired glucose tolerance: a systematic review. Diabet. Med., 2006;6:609-616.

15. Lorenz, MW, Markus, HS, Bots, ML, Rosvall, M, Sitzer, M. Prediction of clinical cardiovascular events with carotid intima-media thickness: a systematic review and metaanalysis. Circulation, 2007;4:459-467. 
16. Wijkman, M, Lanne, T, Engvall, J, Lindstrom, T, Ostgren, CJ, Nystrom, FH. Masked nocturnal hypertension--a novel marker of risk in type 2 diabetes. Diabetologia, 2009;7:12581264.

17. Dahlen, EM, Lanne, T, Engvall, J, Lindstrom, T, Grodzinsky, E, Nystrom, FH et al. Carotid intima-media thickness and apolipoprotein B/apolipoprotein A-I ratio in middle-aged patients with Type 2 diabetes. Diabet. Med., 2009;4:384-390.

18. Fredriksson, I, Larsson, M, Nystrom, FH, Lanne, T, Ostgren, CJ, Stromberg, T. Reduced arteriovenous shunting capacity after local heating and redistribution of baseline skin blood flow in type 2 diabetes assessed with velocity-resolved quantitative laser Doppler flowmetry. Diabetes, 2010;7:1578-1584.

19. Devereux, RB, Reichek, N. Echocardiographic determination of left ventricular mass in man. Anatomic validation of the method. Circulation, 1977;4:613-618.

20. Rosero, EB, Kane, K, Clagett, GP, Timaran, CH. A systematic review of the limitations and approaches to improve detection and management of peripheral arterial disease in Hispanics. J. Vasc. Surg., 2010;4 Suppl:27S-35S.

21. Sigvant, B, Wiberg-Hedman, K, Bergqvist, D, Rolandsson, O, Andersson, B, Persson, E et al. A population-based study of peripheral arterial disease prevalence with special focus on critical limb ischemia and sex differences. J. Vasc. Surg., 2007;6:1185-1191.

22. Yang, X, Sun, K, Zhang, W, Wu, H, Zhang, H, Hui, R. Prevalence of and risk factors for peripheral arterial disease in the patients with hypertension among Han Chinese. J. Vasc. Surg., 2007;2:296-302.

23. Bianchi, C, Penno, G, Pancani, F, Civitelli, A, Piaggesi, A, Caricato, F et al. Nontraditional cardiovascular risk factors contribute to peripheral arterial disease in patients with type 2 diabetes. Diabetes Res. Clin. Pract., 2007;2:246-253.

24. Coutinho, T, Turner, ST, Kullo, IJ. Aortic pulse wave velocity is associated with measures of subclinical target organ damage. JACC Cardiovasc. Imaging, 2011;7:754-761.

25. Lind, L. Arterial stiffness, but not endothelium-dependent vasodilation, is related to a low Ankle-Brachial index. The Prospective Investigation of the Vasculature in Uppsala Seniors (PIVUS). Clin. Physiol. Funct. Imaging, 2011;3:182-187.

26. Poulsen, MK, Henriksen, JE, Dahl, J, Johansen, A, Moller, JE, Gerke, O et al. Myocardial ischemia, carotid, and peripheral arterial disease and their interrelationship in type 2 diabetes patients. J. Nucl. Cardiol., 2009;6:878-887.

27. Wattanakit, K, Folsom, AR, Selvin, E, Coresh, J, Hirsch, AT, Weatherley, BD. Kidney function and risk of peripheral arterial disease: results from the Atherosclerosis Risk in Communities (ARIC) Study. J. Am. Soc. Nephrol., 2007;2:629-636.

28. Sheen, YJ, Lin, JL, Lee, IT, Hsu, YN, Li, TC, Sheu, WH. Low estimated glomerular filtration rate is a major determinant of low ankle-brachial index and toe-brachial index in type 2 diabetes. Angiology, 2012;1:55-61. 
29. Sahli, D, Eliasson, B, Svensson, M, Blohme, G, Eliasson, M, Samuelsson, P et al. Assessment of toe blood pressure is an effective screening method to identify diabetes patients with lower extremity arterial disease. Angiology, 2004;6:641-651.

30. Wu, CK, Yang, CY, Tsai, CT, Chiu, FC, Huang, YT, Lee, JK et al. Association of low glomerular filtration rate and albuminuria with peripheral arterial disease: the National Health and Nutrition Examination Survey, 1999-2004. Atherosclerosis, 2010;1:230-234.

31. Wattanakit, K, Folsom, AR, Criqui, MH, Kramer, HJ, Cushman, M, Shea, S et al. Albuminuria and peripheral arterial disease: results from the multi-ethnic study of atherosclerosis (MESA). Atherosclerosis, 2008;1:212-216. 
Legends

Table 1. Descriptive data on patients with type 2 diabetes and controls.

Table 2. Association between TBI and central arterial PVW, TBI and IMT and as well as TBI and carotid plaque.

Figure 1. Descriptive data and vascular measures. Correlation coefficient between toe brachial index and age, diabetes duration, sex, waist measure, BMI and different vascular measures. $* *=p$ value $<0.01, * * *=$ p value $<0.001$. TBI $=$ toe brachial index, $\mathrm{BMI}=$ body mass index, $\mathrm{LVMI}=$ left ventricular mass index, $\mathrm{PWVcf}=$ pulse wave velocity from A.Carotis to A.Femoralis, $\mathrm{PWV} c r=$ pulse wave velocity from A.Carotis to A.Radialis, IMT=carotid intima media thickness.

Figure 2. Cardiovascular events. Toe brachial index (mean \pm SEM) in patients with type 2 diabetes with (grey) and without (dark grey) hypertension, angina, heart failure, atrial fibrillation, previous myocardial infarction (MI), stroke, PCI/bypass surgery or any previous cardiovascular events (including MI, stroke, PCI/bypass surgery or angina), respectively. $* *=$ p value $<0.01$ and $* * *=p$ value $<0.001$.

Figure 3. Laborative findings. Correlation coefficient between toe brachial index and laborative measures. $* *=$ p value $<0.01, * * *=p$ value $<0.001$. GFR $=$ estimated glomerular filtration rate (according to Cockcroft-Gault). 
Table 1. Descriptive data on patients with type 2 diabetes and controls.

Sex (Females:males)

Diabetes duration (years)

Age (years)

BMI $\left(\mathrm{kg} / \mathrm{m}^{2}\right)$

TBI

LVMI $\left(\mathrm{g} / \mathrm{m}^{2}\right)$
PWVef $(\mathrm{m} / \mathrm{s})$
PWVer $(\mathrm{m} / \mathrm{s})$
Carotid IMT $(\mathrm{mm})$
Carotid Plaque $(\%$ of individuals $)$

Triglycerides ( $\mathrm{mmol} / \mathrm{l})$

Cholesterol (mmol/l)

HDL-Cholesterol (mmol/l)

LDL-Cholesterol (mmol/l)

HDL/LDL

Non-HDL

APO A1 (g/l)

APO B (g/l)

HbA1c -MonoS (\%)

HbA1c - IFCC (mmol/mol)

Potassium (mmol/l)

$\operatorname{GFR}\left(\mathrm{ml} / \mathrm{min} / \mathbf{1 . 7 3 m ^ { 2 }}\right)$

Clinical caracteristics

Previous ischemic event* Angina

Atrial fibrilation

Previous myocardial infarction

Previous stroke

Previous PCI/bypass

Medication

AH drugs

$\beta$-blockers

Thiazide diuretics

Loop diuretics

ACE-I/ARB

Calcium channel blockers

Statins

Acetylsalicylic acid

\begin{tabular}{|c|c|c|}
\hline \multicolumn{2}{|c|}{ Mean (_SD) } & \multirow{3}{*}{$\begin{array}{c}\text { Controls vs DM } \\
p<0.001\end{array}$} \\
\hline Type 2 diabetes & Controls & \\
\hline $482: 260$ & $80: 75$ & \\
\hline $7.2 \pm 6.2$ & & \\
\hline $60.7 \pm 3.1$ & $61.1 \pm 5.2$ & \\
\hline $30.1 \pm 4.7$ & $26.0 \pm 3.4$ & $p<0.001$ \\
\hline $0.81 \pm 0.14$ & $0.87 \pm 0.15$ & $p<0.001$ \\
\hline $120.6 \pm 36.6$ & $116.3 \pm 29.8$ & \\
\hline $10.4 \pm 2.2$ & $9.3 \pm 2.3$ & $p<0.001$ \\
\hline $9.0 \pm 2.5$ & $8.9 \pm 1.2$ & \\
\hline $0.73 \pm 0.2$ & $0.69 \pm 0.2$ & $p<0.05$ \\
\hline $68 \%$ & $43 \%$ & $p<0.001$ \\
\hline $1.8 \pm 1.1$ & $1.3 \pm 0.7$ & $p<0.001$ \\
\hline $4.7 \pm 1.0$ & $5.8 \pm 1.0$ & $p<0.001$ \\
\hline $1.3 \pm 0.3$ & $1.6 \pm 0.5$ & $p<0.001$ \\
\hline $2.7 \pm 0.8$ & $3.5 \pm 0.9$ & $p<0.001$ \\
\hline $2.2 \pm 0.8$ & $2.4 \pm 0.9$ & $p<0.01$ \\
\hline $3.4 \pm 0.9$ & $3.1 \pm 0.9$ & $p<0.001$ \\
\hline $1.3 \pm 0.2$ & & \\
\hline $0.96 \pm 0.2$ & & \\
\hline $6.1 \pm 1.1$ & & \\
\hline $53 \pm 1$ & & \\
\hline $4.1 \pm 0.4$ & & \\
\hline $101 \pm 27.0$ & & \\
\hline
\end{tabular}

\begin{tabular}{ccc}
$15 \%$ & $8 \%$ & $p<0.05$ \\
$8 \%$ & $1 \%$ & $p<0.01$ \\
$2 \%$ & $5 \%$ & $p<0.05$ \\
$9 \%$ & $4 \%$ & \\
$3 \%$ & $2 \%$ & \\
$7 \%$ & $3 \%$ & \\
& & \\
$65 \%$ & $25 \%$ & $p<0.001$ \\
$35 \%$ & $15 \%$ & $p<0.001$ \\
$10 \%$ & $8 \%$ & \\
$8 \%$ & $0 \%$ & $p<0.05$ \\
$43 \%$ & $13 \%$ & $p<0.001$ \\
$15 \%$ & $6 \%$ & $p<0.05$ \\
$55 \%$ & $11 \%$ & $p<0.001$ \\
$29 \%$ & $9 \%$ & $p<0.001$ \\
\hline
\end{tabular}


BMI Body Mass Index (weight/height ${ }^{2}$ )

TBI Toe Brachial Index (TP/Brachial SBP)

LVMI Left Ventricular Mass Index

PWVcf Pulse wave velocity A.Carotis to A.Femoralis

PWVcr Pulse wave velocity A.Carotis to A.Radialis

IMT Intima Media Thickness

GFR Glomerular Filtration Rate (according to Cockcroft-Gault )

*Previous ischemic event $=$ myocardial or stroke, $\mathrm{PCI} /$ bypass or angina

ACE-I angiotensin-converting enzyme inhibitor

$\mathrm{ARB}$ angiotensin receptor blockers 
Table 2. Association between TBI and central arterial PVW, TBI and IMT and as well as TBI and carotid plaque.

\begin{tabular}{|c|c|c|c|c|}
\hline Variable & $\begin{array}{l}\text { B (PWV and } \\
\text { IMT) } \\
\text { OR (Plaque) }\end{array}$ & \multicolumn{2}{|c|}{$\begin{array}{c}95.0 \% \text { Confidence Interval } \\
\text { for B/OR }\end{array}$} & P-value \\
\hline PWV model1* & -1.418 & $(-2.550$ & $-\quad-0.287)$ & 0.014 \\
\hline PWV model2* & -1.366 & $(-2.515$ & $-\quad-.0217)$ & 0.020 \\
\hline PWV model3* & -1.549 & $(-2.717$ & $--0.381)$ & 0.009 \\
\hline IMT model1* & -0.150 & $(-0.244$ & $-\quad-0.056)$ & 0.002 \\
\hline IMT model2* & -0.147 & $(-0.243$ & $--0.052)$ & 0.003 \\
\hline IMT model3* & -0.175 & $(-0.274$ & $--0.077)$ & 0.001 \\
\hline Plaque model1 $1^{\#}$ & 0.175 & $(0.053$ & $-0.576)$ & 0.004 \\
\hline Plaque model $2^{\#}$ & 0.187 & $(0.055$ & $-0.636)$ & 0.007 \\
\hline Plaque model $3^{\#}$ & 0.166 & $(0.047$ & $-0.591)$ & 0.006 \\
\hline
\end{tabular}

*Linear regression regarding PWV and IMT

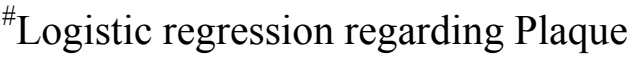

Model 1 Age and diabetes duration

Model 2 Model $1+$ angina, stroke, myocardial infarction, heart failure and atrial fibrillation

Model 3 Model $2+$ glomerular filtration rate

PWV: Pulse wave velocity from A.Carotis to A.Femoralis

IMT: Intima Media Thickness

Plaque: Carotid plaque

OR: odds ratio 


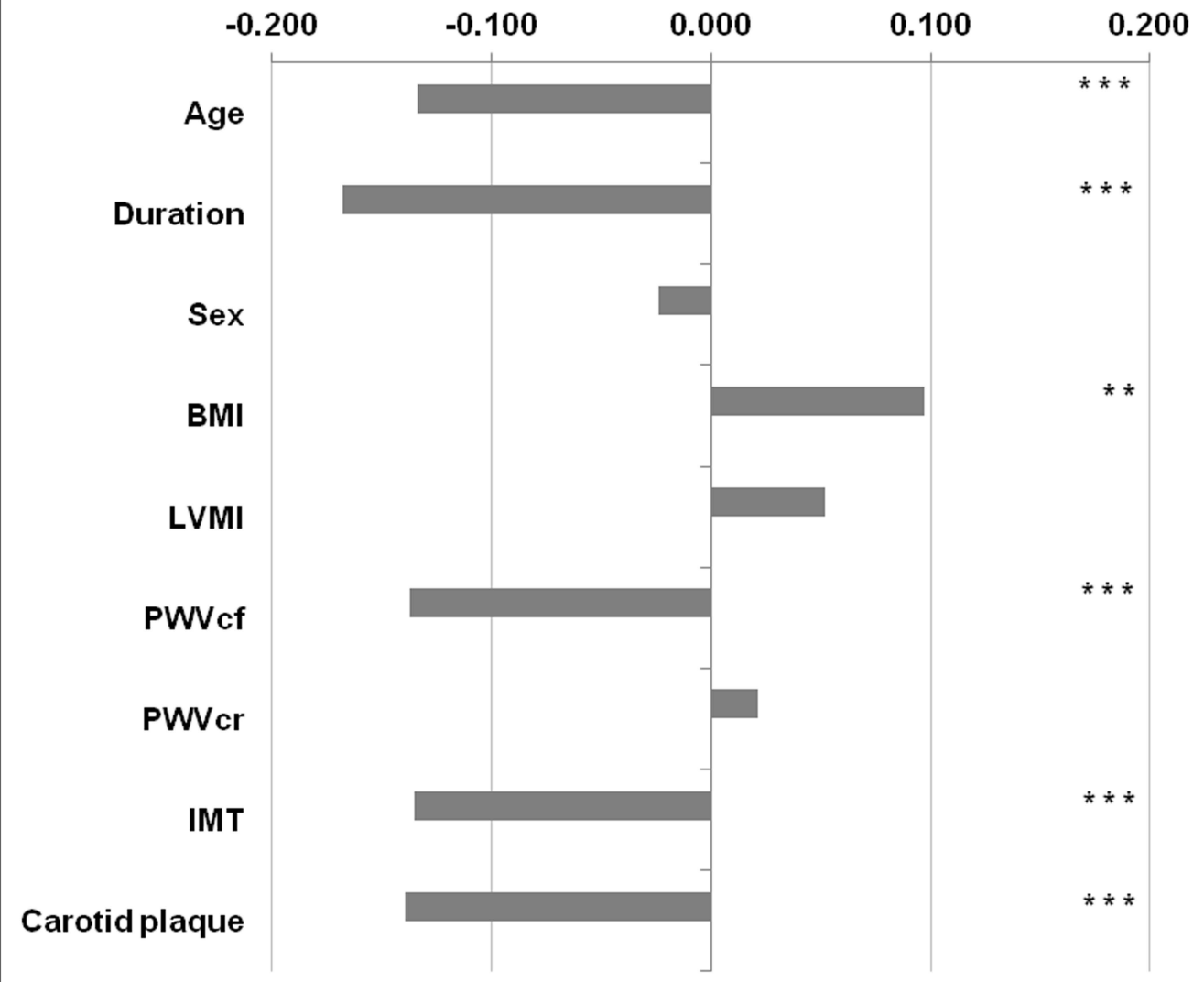




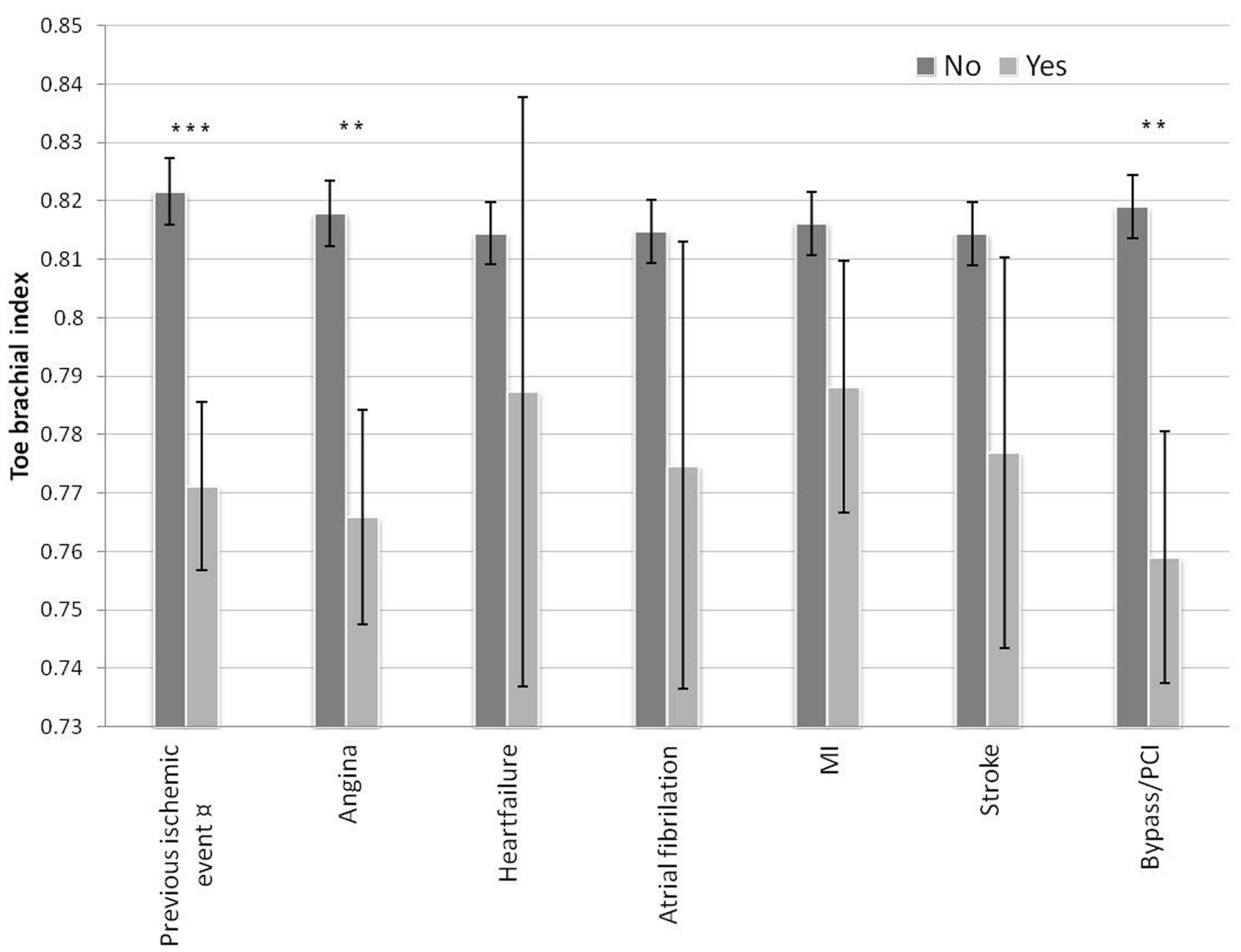




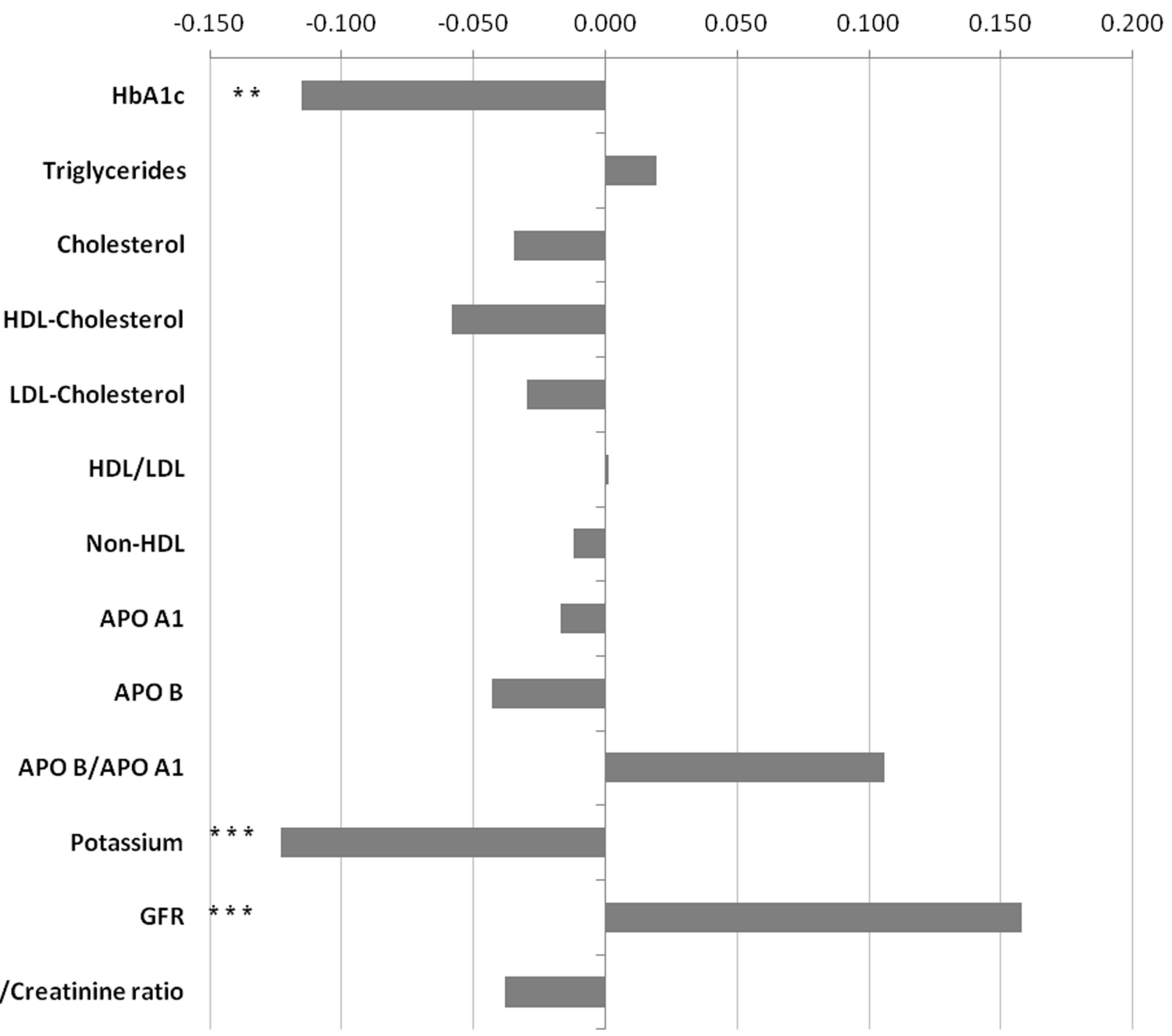

